\title{
A NOTE ON CONVERGENCE THEOREMS FOR INTEGRALS
}

\author{
TUNCAY TUNC AND ALPER ERDEM
}

Received 07 September, 2020

\begin{abstract}
In this study we investigate the convergence theorems such as bounded convergence theorem, Fatou's lemma, monotone convergence theorem and dominated convergence theorem for measurable functions depending upon the concept of statistical convergence.
\end{abstract}

2010 Mathematics Subject Classification: 40G15; 40A35

Keywords: statistically convergence, convergence theorems, Lebesgue integralibility

\section{INTRODUCTION}

When we have a sequence of integrable functions, "the convergence theorems" depend on this sequence state that the integrability is preserved under the operation of limit. More clearly, let $\left(f_{n}\right)$ be a sequence of integrable functions and the function $f$ be some kind of limit of the sequence. Then we naturally desire integralibility of the function $f$ and the relation

$$
\int f=\lim \int f_{n}
$$

holds. We call this type of results as convergence theorems. These theorems are used to show that a given function is integrable or to construct an integrable function. The best known theorem of among the convergence theorems in measure the theory is the Lebesgue dominated convergence theorem.

In this work, we prove the certain convergence theorems in $\sigma-$ finite measurable spaces, when a given sequence of measurable functions is statistically converges to a function.

The concept of statistical convergence was firstly used as "almost convergence" by Zygmund [26] in a monograph in 1935 and in a few years later related works had been published by Steinhaus [25] and Fast [5]. There are a large number of papers related with the statistical convergence and its generalisations in different areas of mathematics have been published in recent years, see [3, 4, 6, 8, 11, 18-20,23].

In [24], Srivastava et al. introduced a new concept of the deferred Nörlund equi-statistical convergence to prove a Korovkin type approximation theorem and demonstrated that a theorem is a non-trivial extension of some well-known Korovkin 
type approximation theorems which were proven by earlier authors. Then, in [14] Parida et al. introduced the notion of equi-statistical convergence, statistical point-wise convergence and statistical uniform convergence in conjunction with the deferred statistical convergence and established a inclusion relation between them.

Srivastava and Et [20] introduced the concepts of lacunary strong summability of order $\alpha$ and lacunary statistical convergence of order $\alpha$ of real-valued functions which are measurable (in the Lebesgue sense) in the interval $(1, \infty)$, and they get some relations between them.

Recently, statistical probability convergence has been a dynamic research area due to the fact that it is more general than the statistical convergence as well as the classical convergence. Moreover, such theory is discussed in the study of Fourier Analysis and Approximation Theory. For more details, see [9,21], and references therein. In [21], Srivastava et al. introduced the notion of statistical probability convergence for sequences of random variables and proved a new Korovkin-type approximation theorem with periodic test functions for a sequence of random variables on a Banach space. In [9], Jena et al. introduced various aspects of statistical convergence for sequences of random variables as well as for sequences of real numbers via deferred Cesàro summability mean and proposed different new Korovkintype approximation results with algebraic test functions for a sequence of random variables on a Banach space. In [22], Srivastava et al. introduced the notion of statistical probability convergence via deferred Nörlund summability mean and proved a new Korovkin-type approximation theorem with algebraic test functions for a sequence of random variables on a Banach space.

In [12] Nath and Tripathy introduced the statistical convergence concepts of complex uncertain sequences: statistical convergence almost surely, statistical convergence in measure, statistical convergence in mean, statistical convergence in distribution and statistical convergence uniformly almost surely sequences of complex uncertain sequences defined by Orlicz function.

Throughout the paper, it is convenient to begin with the definitions and some well-known results which are needed. The set of positive integers will denote, as usual, by $\mathbb{N}$.

Definition 1. For $A \subseteq \mathbb{N}$, we denote the density of $A$ by

$$
d(A)=\lim _{n \rightarrow \infty} \frac{|\{k \in A: k \leq n\}|}{n}
$$

if the limit exists.

Definition 2. Let $\left(a_{n}\right)$ be a sequence of real numbers and $a \in \mathbb{R}$. We say that $\left(a_{n}\right)$ is statistically converges to $a$ if for all $\varepsilon>0$

$$
d\left(\left\{n \in \mathbb{N}:\left|a_{n}-a\right| \geq \varepsilon\right\}\right)=0
$$

and denoted by $a_{n} \stackrel{s t}{\rightarrow} a$ or $s t \lim a_{n}=a$. 
We will use $a_{n} \rightarrow a$ or $\lim a_{n}=a$ for usual limit. It is clear that each convergent real number sequence (in ordinary sense) is statistical convergent, but the inverse is not true. Actually, the relation between the usual convergence and statistical convergence of number sequences can be given as follows. Let

$$
\mathcal{K}:=\{K \subset \mathbb{N}: d(K)=1\} .
$$

Proposition 1 ([2]). If $K_{1}, K_{2} \in \mathcal{K}$ then $K_{1} \cap K_{2} \in \mathcal{K}$.

Proposition 2 ([17]). Let $\left(a_{n}\right)$ be a sequence of numbers and $a \in \mathbb{R}$. $a_{n} \stackrel{\text { st }}{\rightarrow}$ a iff there is a set $K=\left\{k_{1}<k_{2}<\cdots\right\} \in \mathcal{K}$ such that $a_{k_{n}} \rightarrow a$.

There is also a relation between the statistically convergence and arithmetic means of bounded sequences of non-negative real numbers.

Proposition 3 ([5]). Let $\left(a_{n}\right)$ be a bounded sequence of non-negative real numbers. Then

$$
a_{n} \stackrel{s t}{\rightarrow} 0 \quad \text { iff } \quad \frac{1}{n} \sum_{k=1}^{n} a_{k} \rightarrow 0 .
$$

From this proposition we can obtain following corollary.

Corollary 1 ([3]). Let $\left(a_{n}\right)$ be a bounded sequence of real numbers and $a \in \mathbb{R}$. Then

$$
a_{n} \stackrel{s t}{\rightarrow} a \quad \text { iff } \quad \frac{1}{n} \sum_{k=1}^{n}\left|a_{n}-a\right| \rightarrow 0 .
$$

Definition 3 ([7]). Let $\left(a_{n}\right)$ be a sequence of real numbers and $L_{\left(a_{n}\right)}$ and $U_{\left(a_{n}\right)}$ be subsets of $\mathbb{R}$ defined as

$$
L_{\left(a_{n}\right)}=\left\{x \in \mathbb{R}: d\left(\left\{n: a_{n}<x\right\}\right) \neq 0\right\}
$$

and

$$
U_{\left(a_{n}\right)}=\left\{x \in \mathbb{R}: d\left(\left\{n: a_{n}>x\right\}\right) \neq 0\right\},
$$

respectively, then the statistical limit inferior and the statistical limit superior of the sequence $\left(a_{n}\right)$ are defined by

$$
s t \liminf a_{n}= \begin{cases}\inf L_{\left(a_{n}\right)} & L_{\left(a_{n}\right)} \neq \varnothing, \\ +\infty & L_{\left(a_{n}\right)}=\varnothing,\end{cases}
$$

and

$$
s t \lim \sup a_{n}= \begin{cases}\sup U_{\left(a_{n}\right)} & U_{\left(a_{n}\right)} \neq \varnothing, \\ -\infty & U_{\left(a_{n}\right)}=\varnothing,\end{cases}
$$

respectively. 
Theorem 1 ([7]). Let $\left(a_{n}\right)$ be a real number sequence. If st $\liminf a_{n}=\alpha$ is finite then for all $\varepsilon>0$

$$
d\left(\left\{n: a_{n}<\alpha+\varepsilon\right\}\right) \neq 0 \text { and } d\left(\left\{n: a_{n}<\alpha-\varepsilon\right\}\right)=0
$$

hold. Conversely, if (1.1) hold for all $\varepsilon>0$ then st $\liminf a_{n}=\alpha$. Similarly, If st $\lim \sup a_{n}=\beta$ is finite, then for all $\varepsilon>0$

$$
d\left(\left\{n: a_{n}>\beta+\varepsilon\right\}\right)=0 \text { and } d\left(\left\{n: a_{n}>\beta-\varepsilon\right\}\right) \neq 0
$$

hold. Conversely, if (1.2) hold for all $\varepsilon>0$ then st $\lim \sup a_{n}=\beta$.

It is clear that the inequailities

$$
\liminf a_{n} \leq s t \liminf a_{n} \leq s t \lim \sup a_{n} \leq \lim \sup a_{n}
$$

hold for any real number sequence $\left(a_{n}\right)$.

Definition 4 ([7]). Let $\left(a_{n}\right)$ be a real number sequence. If there exists a positive real number M satisfying $d\left(\left\{k:\left|x_{k}\right|>M\right\}\right)=0$ then $\left(a_{n}\right)$ is said to be statistically bounded sequence.

Theorem 2 ([7]). Let $\left(a_{n}\right)$ be a statistically bounded real number sequence. Then $\left(a_{n}\right)$ is statistically convergent iff st $\lim \sup a_{n}=s t \liminf a_{n}$.

Note that, for any sequence of real numbers $\left(a_{n}\right)$, if there exists a set $K=$ $\left\{k_{1}<k_{2}<\cdots\right\} \in \mathcal{K}$ such that $s t \lim \sup a_{k_{n}}=\alpha$ then $s t \lim \sup a_{n}=\alpha$. Similarly, if there exists a set $K=\left\{k_{1}<k_{2}<\cdots\right\} \in \mathcal{K}$ such that st $\liminf a_{k_{n}}=\alpha$ then $s t \liminf a_{n}=\alpha$.

Now let us give the definition of the statistical convergence of sequence of functions by using the definition of statistical convergence of real number sequences. The statistical convergence of sequence of functions was first examined in 1951 by Fast [5]. In recent years, there are many studies on the statistical convergence of function sequences (see $[1,2,8,10,13,16]$ and references therein).

Definition 5. For a set $X$, a sequence $\left(f_{n}\right)$ of real valued sequence of functions on $X$ is said to be statistically convergent to $f$ on $X$ if $f_{n}(x) \stackrel{s t}{\rightarrow} f(x)$ for all $x \in X$ and denoted by $f_{n} \stackrel{s t}{\rightarrow} f$.

Many features about the statistical convergence of the function sequences can be obtained directly with the help of the statistical convergence of the number sequences. However, the Proposition 2, which is very useful in number sequences, is unfortunately not valid in the statistical convergence of function sequences. For a counterexample, it can examined the Example 3.6 in [13]. Thus, the Corollary 3.7 in [2] is unfortunately not valid.

In this work, we investigate the convergence theorems such as bounded convergence theorem, Fatou's lemma, monotone convergence theorem and dominated convergence theorem for measurable functions depending the upon the concept of statistical convergence. 


\section{The Statistical Convergence Theorems}

Let $(X, \mathcal{B}, \mu)$ be a $\sigma$-finite measure space and complete, that is $\mathcal{B}$ is a $\sigma$-algebra of subsets of $X, \mu: \mathcal{B} \rightarrow[0, \infty]$ is a countably additive function with $\mu(\varnothing)=0$ and $\mathcal{B}$ contains all subsets of all sets of measure zero (for details see [15]). For example the Lebesgue measure space $(\mathbb{R}, \mathcal{M}, m)$ is such a space where $\mathcal{M}$ is family of all Lebesgue measurable subsets of $\mathbb{R}$. All examples in this section will be selected in the Lebesgue measure space $(\mathbb{R}, \mathcal{M}, m)$.

Definition 6. For $E \in \mathcal{B}$, a sequence $\left(f_{n}\right)$ of $\mu$-measurable real valued functions on $E$ is said to be statistically convergent to $f$ almost everywhere if $\mu\left(\left\{x \in X: s t \lim f_{n}(x) \neq f(x)\right\}\right)=0$ and denoted by $f_{n} \stackrel{\text { st-a.e. }}{\longrightarrow} f$.

Proposition 4 ([13]). If $\left(f_{n}\right)$ is a sequence of real-valued $\mu$-measurable functions and $f_{n} \stackrel{\text { st-a.e. }}{\longrightarrow} f$ then $f$ is a $\mu$-measurable function .

Let us begin with statement of the statistical version of the bounded convergence theorem.

Theorem 3 (The Bounded Statistical Convergence Theorem). Let $E \in \mathfrak{B}$, $\mu(E)<\infty$ and $\left(f_{n}\right)$ be a sequence of $\mu$-measurable functions that uniformly bounded on the set $E$ (i.e. there exists a number $M \geq 0$ such that $\left|f_{n}(x)\right| \leq M$ holds for all $x \in E$ and $n \in \mathbb{N}$ ). If $f_{n} \stackrel{\text { st }- \text { a.e. }}{\longrightarrow} f$ then

$$
\text { st } \lim \int_{E} f_{n} d \mu=\int_{E} f d \mu
$$

holds.

Proof. Without loss of generality assume that $f_{n} \stackrel{s t}{\rightarrow} f$ on $E$. From Proposition 4, the function $f$ is $\mu$-measurable. For fixed $x \in E$, since $f_{n}(x) \stackrel{s t}{\rightarrow} f(x)$ then there is a set $K=\left\{k_{1}<k_{2}<\cdots\right\} \in \mathcal{K}$ such that $\lim _{k \rightarrow \infty} f_{n_{k}}(x)=f(x)$. Hence $|f(x)| \leq M$ holds. Therefore $\left|f_{n}(x)-f(x)\right| \leq 2 M$ for all $x \in E$ and $n \in \mathbb{N}$. By using Corollary 1, we have

$$
\lim _{n \rightarrow \infty} \frac{1}{n} \sum_{k=1}^{n}\left|f_{n}(x)-f(x)\right|=0 \quad \text { for all } \quad x \in E .
$$

If we take $g_{n}:=\frac{1}{n} \sum_{k=1}^{n}\left|f_{n}-f\right|$, then it is clear that $g_{n}$ is $\mu$-measurable and $\left|g_{n}\right| \leq 2 M$ for all $n \in \mathbb{N}$, and $g_{n} \rightarrow 0$. By the Lebesgue Convergence Theorem [15]

$$
\lim _{n \rightarrow \infty} \int_{E} g_{n} d \mu=0 .
$$

Therefore, we have

$$
\lim _{n \rightarrow \infty} \frac{1}{n} \sum_{k=1}^{n} \int_{E}\left|f_{n}-f\right| d \mu=0 .
$$


Considering $\int_{E}\left|f_{n}-f\right| d \mu \geq 0$ for all $n \in \mathbb{N}$ then, by Proposition 3, we obtain

$$
\text { st } \lim _{n \rightarrow \infty} \int_{E}\left|f_{n}-f\right| d \mu=0 \text {. }
$$

From the inequality

$$
\left|\int_{E} f_{n} d \mu-\int_{E} f d \mu\right| \leq \int_{E}\left|f_{n}-f\right| d \mu
$$

we get (2.1) as desired.

Remark 1. In the equatiliy (2.1) we can not substitue st lim with ordinary limit.

Example 1. Let $E=[0,1] \subset \mathbb{R}$ and $f_{n}: E \rightarrow \mathbb{R}, n \in \mathbb{N}$, be defined as

$$
f_{n}(x)= \begin{cases}0 & \text { if } n \text { is square } \\ 1 & \text { if } n \text { is non-square }\end{cases}
$$

It is clear that

$$
f_{n} \stackrel{s t}{\rightarrow} f \equiv 1 \quad \text { and } \quad s t \lim \int_{E} f_{n} d m=\int_{E} f d m=1
$$

but $\lim _{n \rightarrow \infty} \int_{E} f_{n} d m$ does not exist.

Let $f$ and $g$ be a real-valued functions which are defined on $\mu$-measurable set $\mathrm{E}$. Let us define the function

$$
(f \wedge g)(x)=\min \{f(x), g(x)\}, \quad x \in E .
$$

It is easy to see that if $f$ and $g$ are $\mu$-measurable functions then $f \wedge g$ is a $\mu$-measurable function. By using the relation

$$
f \wedge g=\frac{f+g-|f-g|}{2}
$$

we have the following proposition.

Proposition 5. $f_{n} \stackrel{s t}{\rightarrow} f$ and $g_{n} \stackrel{s t}{\rightarrow} g$ then $f_{n} \wedge g_{n} \stackrel{s t}{\rightarrow} f \wedge g$.

Let $E \in \mathcal{B}$. Let us denote the family of measurable functions by $\mathcal{L}_{0}(E ; \mu)$ and the family of positive measurable functions by $\mathcal{L}_{0}^{+}(E ; \mu)$.

Definition 7. For a sequence $\left(f_{n}\right) \subset \mathcal{L}_{0}(E ; \mu)$, if there exists a set $K=$ $\left\{k_{1}<k_{2}<\cdots\right\} \in \mathcal{K}$ such that $\left(f_{k_{n}}\right)_{n} \subset \mathcal{L}_{0}^{+}(E ; \mu)$ then the sequence $\left(f_{n}\right)$ is said to be statistically positive on $E$.

For example the sequence $\left(f_{n}\right)$ defined on $\mathbb{R}$ as

$$
f_{n}(x)= \begin{cases}-1 & \text { if } n \text { is prime } \\ x^{2} / n & \text { otherwise }\end{cases}
$$

is statistically positive on $\mathbb{R}$. 
The statistical version of the Fatou's Lemma which is handy to use when proving the convergence theorems is given below.

Theorem 4. Let $\left(f_{n}\right) \subset \mathcal{L}_{0}^{+}(E ; \mu)$ be a statistically positive sequence of functions and $f_{n} \stackrel{\text { st-a.e. }}{\longrightarrow} f$ then

$$
\int_{E} f d \mu \leq s t \liminf _{n} \int_{E} f_{n} d \mu
$$

holds.

Proof. Without loss of generality, assume that $f_{n} \stackrel{s t}{\rightarrow} f$ and $\left(f_{n}\right) \subset \mathcal{L}_{0}^{+}(E ; \mu)$. It can be easily seen that $f \in \mathcal{L}_{0}^{+}(E ; \mu)$. Take an arbitrary function $h \in \mathcal{L}_{0}(E ; \mu)$ satisfying the following conditions:

(i) $h<f$,

(ii) there exists a number $M \geq 0$ such that $|h| \leq M$,

(iii) for $E_{h}=\{x \in E: h(x) \neq 0\}$, let $\mu\left(E_{h}\right)<\infty$.

Let us consider $h_{n}:=f_{n} \wedge h, n \in \mathbb{N}$. It is clear that, the function $\left(h_{n}\right)$ satisfies the properities $(i),(i i)$ and (iii) for all $n \in \mathbb{N}$. In addition, by Proposition $5, h_{n} \stackrel{s t}{\rightarrow} h$ holds. According to the bounded statistical convergence theorem (Theorem 2.1), we have

$$
\int_{E_{h}} h d \mu=s t \lim \int_{E_{h}} h_{n} d \mu .
$$

Since $h_{n} \leq f_{n}$ for all $n \in \mathbb{N}$ then the inequalities

$$
\int_{E_{h}} h_{n} d \mu \leq \int_{E_{h}} f_{n} d \mu \leq \int_{E} f_{n} d \mu
$$

are valid. Thus, by using (2.3), we get

$$
\int_{E} h d \mu=s t \lim \int_{E} h_{n} d \mu=s t \liminf _{n} \int_{E} h_{n} d \mu \leq s t \liminf _{n} \int_{E} f_{n} d \mu .
$$

By taking supremum on the functions $h$ that satisfy the properities $(i),(i i)$ and (iii), we obtain

$$
\int_{E} f d \mu=\sup _{h} \int_{E} h d \mu \leq s t \liminf _{n} \int_{E} f_{n} d \mu
$$

as desired.

Remark 2. The strict inequality in (2.2) exists and it is not allowed to substitue the st liminf with ordinary liminf.

Example 2. Let $E=[0, \infty)$ and $f_{n}: E \rightarrow \mathbb{R}$ be defined as

$$
f_{n}(x)= \begin{cases}0 & \text { if } n \text { is square, } \\ \frac{\chi_{0}^{n-1}(x)}{(x+1)^{2}}+\frac{n+2}{2 n} \chi_{n-1}^{n}(x) & \text { if } n \text { is even but non-square, } \\ \frac{\chi_{0}^{n-1}(x)}{(x+1)^{2}}+\frac{n+1}{n} \chi_{n-1}^{n}(x) & \text { if } n \text { is odd but non-square, }\end{cases}
$$


where $\chi_{a}^{b}:=\chi_{[a, b]}$ is the characteristic function of the interval $[a, b]$. It is clear that $f_{n} \geq 0$ for all $n \in \mathbb{N}$ and $f_{n}(x) \stackrel{s t}{\rightarrow} f(x)=(x+1)^{-2}$ for all $x \in E$. It can be easily obtained the following strict inequalities

$$
\liminf \int_{E} f_{n} d m=0<\int_{E} f d m=1<s t \liminf \int_{E} f_{n} d m=\frac{3}{2} .
$$

Definition 8. A sequence $\left(f_{n}\right)$ of functions is called statistically increasing if there exists a set $K=\left\{k_{1}<k_{2}<\cdots\right\} \in \mathcal{K}$ such that $f_{k_{n+1}}-f_{k_{n}} \geq 0$ for all $n \in \mathbb{N}$.

The following theorem is the statistical version of the monotone convergence theorem for measurable functions.

Theorem 5 (Monotone Statistical Convergence Theorem). Let $\left(f_{n}\right) \subset \mathcal{L}_{0}(E ; \mu)$ be a statistically increasing and statistically positive sequence of functions. If $f_{n} \stackrel{\text { st }- \text { a.e. }}{\longrightarrow} f$ then

$$
\int_{E} f d \mu=s t \lim _{n \rightarrow \infty} \int_{E} f_{n} d \mu
$$

holds.

Proof. Since the sequence $\left(f_{n}\right)$ is statistically positive and $f_{n} \stackrel{\text { st-a.e. }}{\longrightarrow} f$, by using Theorem 4, we have

$$
\int_{E} f d \mu \leq s t \liminf \int_{E} f_{n} d \mu .
$$

Considering Proposition 1, there is a set $K \in \mathcal{K}$ such that the subsequence $\left(f_{n}\right)_{n \in K}$ is positive and increasing on the set $E$. Let $K=\left\{k_{1}<k_{2}<\cdots\right\}$. Then $f_{k_{n}} \leq f$ for all $n \in \mathbb{N}$. Thus

Thereby

$$
\int_{E} f_{k_{n}} d \mu \leq \int_{E} f d \mu
$$

$$
s t \lim \sup \int_{E} f_{n} d \mu=s t \limsup \int_{E} f_{k_{n}} d \mu \leq \int_{E} f d \mu
$$

holds. By gathering the inequalities (2.5) and (2.6), the inequality (2.4) can be obtained as desired.

Remark 3. In the inequality (2.4) it is not allowed to substitue the st lim with usual limit.

Example 3. Let $E=[0, \infty)$ and $f_{n}: E \rightarrow \mathbb{R}$ be defined as

$$
f_{n}(x)= \begin{cases}-\chi_{0}^{n}(x) / n & \text { if } n \text { is square } \\ \chi_{0}^{n}(x) /(x+1)^{2} & \text { otherwise. }\end{cases}
$$

It is clear that $f_{n} \in \mathcal{L}_{0}[0, \infty)$ for all $n$, and the sequence $\left(f_{n}\right)$ is statistically positive and statistically increasing. In addition, st $\lim f_{n}(x)=(1+x)^{-2}=: f(x)$ for all $x \in[0, \infty)$. 
Let $a_{n}=\int_{E} f_{n} d m, n \in \mathbb{N}$. Then

$$
a_{n}= \begin{cases}-1 & \text { if } n \text { is square } \\ \frac{n}{(n+1)} & \text { otherwise }\end{cases}
$$

Thus, $s t \lim a_{n}=1$ while $\lim a_{n}$ does not exist.

Theorem 6 (Dominated Statistical Convergence Theorem). Let $g$ be a $\mu$-integrable function on $E$ and $\left(f_{n}\right) \subset \mathcal{L}_{0}(E ; \mu)$. If $\left|f_{n}(x)\right| \leq g(x)$ for all $n \in \mathbb{N}$ and $x \in E$ and $f_{n} \stackrel{\text { st }}{\rightarrow} f$ then $f$ is $\mu$-integrable on $E$ and

$$
s t \lim \int_{E} f_{n} d \mu=\int_{E} f d \mu
$$

holds.

Proof. It is clear that $f_{n}$ is $\mu$-integrable function for all $n \in \mathbb{N}$. From assumption, for arbitrary fixed number $x \in E$, st $\lim f_{n}(x)=f(x)$ implies existness of a set $K=$ $\left\{k_{1}<k_{2}<\cdots\right\} \in \mathcal{K}$ such that $\lim f_{k_{n}}(x)=f(x)$. By the fact that $\left|f_{k_{n}}(x)\right| \leq g(x)$, we get $|f(x)| \leq g(x)$ for all $x \in E$. From assumption $g$ is a $\mu$-integrable function then $f$ is $\mu$-ingtegrable function.

Let us consider the functions $h_{n}=g+f_{n}$ and $g_{n}=g-f_{n}, n \in \mathbb{N}$. Then both sequences $\left(h_{n}\right)$ and $\left(g_{n}\right)$ consist from non-negative $\mu$-integrable functions. Since $h_{n} \stackrel{s t}{\rightarrow} g+f$, by Theorem 4, we have

$$
\int_{E}(g+f) d \mu \leq s t \liminf \int_{E}\left(g+f_{n}\right) d \mu
$$

hence

$$
\int_{E} f d \mu \leq s t \liminf \int_{E} f_{n} d \mu
$$

and since $h_{n} \stackrel{s t}{\rightarrow} g+f$, by Theorem 4 , we have

$$
\int_{E}(g-f) d \mu \leq s t \liminf \int_{E}\left(g-f_{n}\right) d \mu
$$

hence

$$
\int_{E} f d \mu \geq s t \limsup \int_{E} f_{n} d \mu .
$$

Combining (2.8) and (2.9) we obtain (2.7) as desired.

Definition 9 ([16]). Let $\left(f_{n}\right)$ be a sequence of functions and $g$ be a function defined on $E$. If

$$
d\left(\left\{n:\left|f_{n}(x)\right| \leq g(x), \forall x \in E\right\}\right)=0
$$

then $g$ is called a statistically majorant function for the sequence $\left(f_{n}\right)$. 
Corollary 2. $g \in \mathcal{L}_{1}(E ; \mu)$ and $\left(f_{n}\right) \in \mathcal{L}_{0}(E ; \mu)$ is given. If the function $g$ is a statistically majorant function for the sequence $\left(f_{n}\right)$ and $f_{n} \stackrel{\text { st-a.e. }}{\longrightarrow} f$ then

$$
\text { st } \lim \int_{E} f_{n} d \mu=\int_{E} f d \mu .
$$

It can not be applied the statistical versions of bounded, monotone and uniform convergence theorems to the following example, but Corollary 2 can be applied. This example also shows that it can not be replaced the $s t$ lim with usual limit in (2.10).

Example 4. Let $E=[0,2]$ and $f_{n}: E \rightarrow \mathbb{R}$ be defined as

$$
f_{n}(x)= \begin{cases}n & \text { if } n \text { is square } \\ \sqrt{n} & \text { if } n \text { is non-square and } 1 / n \leq x \leq 2 / n, \\ 0 & \text { otherwise }\end{cases}
$$

The function $g$ defined as $g(x)=\sqrt{2 / x}$ is statistically mojorant for the sequence $\left(f_{n}\right)$.

\section{REFERENCES}

[1] M. Balcerzak, K. Dems, and A. Komisarski, "Statistical convergence and ideal convergence for sequences of functions," J. Math. Anal. Appl., vol. 328, no. 1, pp. 715-729, 2007, doi: 10.1016/j.jmaa.2006.05.040.

[2] B. T. Bilalov and T. Y. Nazarova, "Statistical convergence of functional sequences," Rocky Mt. J. Math., vol. 45, no. 5, pp. 1413-1423, 2015, doi: 10.1216/RMJ-2015-45-5-1413.

[3] J. S. Connor, "The statistical and strong p-Cesàro convergence of sequences," Analysis , vol. 8, no. 1-2, pp. 47-63, 1988, doi: 10.1524/anly.1988.8.12.47.

[4] P. Erdös and P. Tenenbaüm, "Sur les densités de certaines suites d'entiers," Proc. Lond. Math. Soc. (3), vol. 59, no. 3, pp. 417-438, 1989, doi: 10.1112/plms/s3-59.3.417.

[5] H. Fast, "Sur la convergence statistique," Colloq. Math., vol. 2, pp. 241-244, 1951, doi: 10.4064/cm-2-3-4-241-244.

[6] J. A. Fridy, "On statistical convergence," Analysis, vol. 5, pp. 301-313, 1985, doi: 10.1524/anly.1985.5.4.301.

[7] J. A. Fridy and C. Orhan, "Statistical limit superior and limit inferior," Proc. Am. Math. Soc., vol. 125, no. 12, pp. 3625-3631, 1997, doi: 10.1090/S0002-9939-97-04000-8.

[8] A. D. Gadjiev and C. Orhan, "Some approximation theorems via statistical convergence," Rocky Mt. J. Math., vol. 32, no. 1, pp. 129-138, 2002, doi: 10.1216/rmjm/1030539612.

[9] B. B. Jena, S. K. Paikray, and H. Dutta, "On various new concepts of statistical convergence for sequences of random variables via deferred Cesàro mean," J. Math. Anal. Appl., vol. 487, no. 1, p. 18, 2020, id/No 123950, doi: 10.1016/j.jmaa.2020.123950.

[10] O. Kisi and E. Güler, "On statistical convergence with respect to measure," J. Class. Anal., vol. 10, no. 1 , pp. 77-85, 2017, doi: 10.7153/jca-10-08.

[11] H. I. Miller, "A measure theoretical subsequence characterization of statistical convergence," Trans. Am. Math. Soc., vol. 347, no. 5, pp. 1811-1819, 1995, doi: 10.1090/S0002-9947-19951260176-6.

[12] P. K. Nath and B. C. Tripathy, "Statistical convergence of complex uncertain sequences defined by Orlicz function," Proyecciones, vol. 39, no. 2, pp. 301-315, 2020, doi: 10.22199/issn.07176279-2020-02-0019. 
[13] C. Papachristodoulos, "The classical theorems of measure theory in connection with the statistical convergence and some remarks on Steinhaus' theorem," theorem, arXiv preprint arXiv: 1702.07154.

[14] P. Parida, S. K. Paikray, and B. B. Jena, "Generalized deferred Cesàro equi-statistical convergence and analogous approximation theorems," Proyecciones, vol. 39, no. 2, pp. 317-339, 2020, doi: 10.22199/issn.0717-6279-2020-02-0020.

[15] H. L. Royden, Real analysis., 3rd ed. Macmillan New York, 1988.

[16] S. R. Sadigova and T. Y. Nazarova, "Statistical type Lebesgue and Riesz theorems," International Journal of Mathematical Analysis, vol. 34, no. 9, p. 1669-1683, 2015, doi: 10.12988/ijma.2015.53122.

[17] T. Šalát, "On statistically convergent sequences of real numbers," Math. Slovaca, vol. 30, pp. $139-150,1980$.

[18] I. J. Schoenberg, "The integrability of certain functions and related summability methods. I, II," Am. Math. Mon., vol. 66, pp. 361-375, 562-563, 1959, doi: 10.1080/00029890.1959.11989303.

[19] H. M. Srivastava, N. L. Braha, and V. L. and, "Lambda 2-weighted statistical convergence and Korovkin and Voronovskaya type theorems," Appl. Math. Comput., vol. 266, pp. 675-686, 2015, doi: 10.1016/j.amc.2015.05.108.

[20] H. M. Srivastava and M. Et, "Lacunary statistical convergence and strongly Lacunary summable functions of order alpha," Filomat, vol. 31, pp. 1573-1582, doi: 10.2298/FIL1706573S.

[21] H. M. Srivastava, B. B. Jena, and S. K. Paikray, "A certain class of statistical probability convergence and its applications to approximation theorems," Applicable Analysis and Discrete Mathematics, vol. 14, pp. 579-598J, doi: 10.2298/AADM190220039S.

[22] H. M. Srivastava, B. B. Jena, and S. K. Paikray, "Statistical probability convergence via the deferred Nörlund mean and its applications to approximation theorems," Rev. R. Acad. Cienc. Exactas Fís. Nat., Ser. A Mat., RACSAM, vol. 114, no. 3, p. 14, 2020, id/No 144, doi: 10.1007/s13398-020-00875-7.

[23] H. M. Srivastava, B. B. Jena, S. K. Paikray, and U. K. Misra, “A certain class of weighted statistical convergence and associated Korovkin-type approximation theorems involving trigonometric functions," Mathematical Methods in the Applied Sciences, vol. 41, no. 2, pp. 671-683, 2018, doi: 10.1002/mma.4636.

[24] H. M. Srivastava, B. B. Jena, S. K. Paikray, and U. K. Misra, "Generalized equi-statistical convergence of the deferred Nörlund summability and its applications to associated approximation theorems," Rev. R. Acad. Cienc. Exactas Fís. Nat., Ser. A Mat., RACSAM, vol. 112, no. 4, pp. 1487-1501, 2018, doi: 10.22199/issn.0717-6279-2020-02-0020.

[25] H. Steinhaus, "Sur la convergence ordinarie et la convergence asymptotique," Colloq. Math., vol. 2, no. 1, pp. 73-74, 1951, doi: 10.4064/cm-2-1-63-78.

[26] A. Zygmund, “Trigonometric series, 2nd ed." Cambridge: At the University Press, 1959.

\section{Authors' addresses}

Tuncay Tunc

Mersin University, Department of Mathematics, Ciftlikköy Yerleskesi, 33110 Mersin, Turkey

E-mail address: ttuncemersin.edu.tr

\section{Alper Erdem}

(Corresponding author) Mersin University, Department of Mathematics, Ciftlikköy Yerleskesi, 33110 Mersin, Turkey

E-mail address: alpererdememersin.edu.tr 